\title{
Nonlinear pattern selection in a mechanical model for morphogenesis
}

\author{
A. S. Perelson ${ }^{1}$, P. K. Maini ${ }^{2}$, J. D. Murray ${ }^{2}$, J. M. Hyman ${ }^{1}$ and G. F. Oster ${ }^{3}$ \\ ${ }^{1}$ Theoretical Division, Los Alamos National Laboratory, Los Alamos, NM 87545, USA \\ ${ }^{2}$ Centre for Mathematical Biology, Mathematical Institute, University of Oxford, \\ Oxford OX1 3LB, U.K. \\ ${ }^{3}$ Departments of Biophysics and Entomology, University of California, Berkeley, CA 94720, USA
}

\begin{abstract}
We present a numerical study of the nonlinear mechanical model for morphogenesis proposed by Oster et al. (1983) with the aim of establishing the pattern forming capability of the model. We present a technique for mode selection based on linear analysis and show that, in many cases, it is a reliable predictor for nonlinear mode selection. In order to determine the set of model parameters that can generate a particular pattern we develop a technique based on nonlinear least square fitting to a dispersion relation. As an application we present a scenario for sequential pattern formation of dermal aggregations in chick embryos which leads to the hexagonal array of cell aggregations observed in feather germ formation in vivo.
\end{abstract}

\section{Mechanical model and background}

A central issue in developmental biology is the formation of spatial pattern and form in the early embryo. Various models and mechanisms have been proposed to describe the pattern formation process, such as those based on the reactiondiffusion theory of Turing (1952) involving chemicals called morphogens (see, for example, Gierer and Meinhardt (1972), Murray $(1977,1981)$ and Meinhardt (1983)). Such models can produce various patterns observed in development; the existence of morphogens in embryology, however, has yet to be established.

An alternative approach to morphogenetic pattern formation is based on the mechanochemical interaction of mesenchymal cells with the extracellular matrix (ECM) - a complex of cross-linked collagen fibres and glycosaminoglycans (GAGS) - on which they move (see, for example, Oster et al. (1983), Murray and Oster (1984a, b), Oster et al. (1985)). This new approach to pattern formation has been applied to several widely studied phenomena in developmental biology, such as the formation of feather germ primordia in chicks (Sengel (1976), Davidson (1983a, b), Dhouailly (1983)) and cartilage formation in chick and amphibian limbs (for example, Wolpert (1981), Maden (1982), Solursh (1983), Shubin and Alberch (1986)). 
In this paper, by studying nonlinear pattern selection we extend the predictions of possible spatial patterns in the mechanical model proposed by Oster et al. (1983). Their analysis, based on linear theory, indicated that stable spatial patterns would evolve from the full nonlinear system of equations. Here we explicitly demonstrate a set of such patterns obtained in a variety of parameter regimes. A key aspect of this paper is the technique used for pattern selection. When a dispersion relation qualitatively indicates linear growth of a particular wave number, the selection of the specific parameter values to isolate such a wave number is not a trivial exercise. This is particularly so when the number of parameters involved is more than one or two, as is the case with these mechanochemical models. Here we develop a technique based on nonlinear least squares fitting to a desired dispersion relation that allows us to isolate particular wave numbers when the number of parameters is large. We study an example involving seven parameters. To set the stage for our analysis, we give a brief introduction to the model equations and refer the reader to the original paper (Oster et al. (1983)) for fuller details.

The model consists of conservation equations for mesenchymal cell and extracellular matrix (ECM) densities, $n(x, t)$ and $\rho(x, t)$ respectively, where $x$ is the spatial coordinate and $t$ the time. These are coupled with a force balance equation for the mechanical interaction of cells with the matrix, which determines the displacement, $u(x, t)$, of a material point of ECM located at position $x$. We consider each equation in turn.

\section{Cell conservation equation}

The equation for cell density is of the form

$$
\partial n / \partial t=-\nabla \cdot \underline{J}_{n}+\text { cell division }
$$

where $\underline{J}_{n}$ is the cell flux, which consists of several terms:

Diffusion. We take the flux due to random motion to be given by

$$
\underline{J}_{\text {diftusion }}=-D_{1} \nabla n \text {. }
$$

For simplicity we take $D_{1}$ to be constant. Oster et al. (1983) include a long range diffusional flux, which is not included here again for simplicity.

Haptotaxis. Cells move by attaching their lamellapodia to specialized adhesive sites in the ECM. The tendency of cells to move up a gradient in adhesive site density is known as haptotaxis and is modelled by

$$
\underline{J}_{\text {haptotaxis }}=\alpha n \nabla \rho,
$$

where $\alpha$ is a non-negative constant.

Convection. Cells may be convected by the motion of the matrix with the convective velocity $\partial \underline{u} / \partial t$. Thus,

$$
\underline{J}_{\text {convection }}=n \partial \underline{u} / \partial t .
$$

Therefore, the total flux, $\underline{J}_{n}$, is given by

$$
\underline{J}_{n}=\underline{J}_{\text {diffusion }}+\underline{J}_{\text {haptotaxis }}+\underline{J}_{\text {convection }} \text {. }
$$


Cell division. Cell division (mitosis) increases sigmoidally with cell density, and so we qualitatively model this with the simplest term exhibiting such sigmoidal behaviour, namely the logistic form

$$
\text { mitotic rate }=r n(N-n)
$$

where $r$ and $N$ are positive constants.

Substituting (1.2) and (1.3) into (1.1) gives the equation for cell conservation.

\section{Force balance equation}

As we are dealing with a system with low Reynolds number, inertia terms may be neglected, and the stress tensor $\sigma(x, t)$ satisfies the force balance equation

$$
\nabla \cdot \boldsymbol{\sigma}+\rho \underline{F}=\underline{\mathbf{0}}
$$

where $F$ is an external body force (Purcell (1977)). The stress tensor, $\boldsymbol{\sigma}$, consists of two components,

$$
\boldsymbol{\sigma}=\boldsymbol{\sigma}_{\text {matrix }}+\boldsymbol{\sigma}_{\text {cell-matrix }}
$$

We model the matrix as a linear, isotropic, visco-elastic material with stress tensor

$$
\begin{gathered}
\boldsymbol{\sigma}_{\text {matrix }}=\mu_{1} \partial \boldsymbol{\varepsilon} / \partial t+\mu_{2} \partial \theta / \partial t \mathbf{I}+[E /(1+\nu)][\boldsymbol{\varepsilon}+\nu \theta \mathbf{I} /(1-2 \nu)] \\
\text { viscous }
\end{gathered}
$$

where $E$ is Young's modulus, $\mu_{1}$ and $\mu_{2}$ the shear and bulk viscosities respectively, $\nu$ the Poisson ratio, $\theta=\nabla \cdot \underline{u}$, the dilatation, $\varepsilon=\frac{1}{2}\left(\nabla \cdot \underline{u}+\nabla \cdot \underline{u}^{T}\right)$ is the (linear) strain, and $I$ is the unit tensor.

Mesenchymal cells can exert large traction forces by attaching to the matrix (Harris et al. 1980). We model the stress due to this cell-matrix interaction by

$$
\boldsymbol{\sigma}_{\text {cell-matrix }}=\tau(n) n\left[\rho+\beta \nabla^{2} \rho\right] \mathbf{I},
$$

where $\tau(n)=\tau /\left(1+\lambda n^{p}\right), p \geqslant 2$, is the traction per unit length per cell, and $\tau, \lambda$ and $\beta$ are positive constants. The $\beta \nabla^{2} \rho$ term accounts for long range traction, the inclusion of which is required because of the fibrous nature of the ECM. The form of $\tau(n)$ takes into account contact inhibition: as cell density increases, cells come into contact with each other and their motion is inhibited.

We assume that the cell-matrix composite is attached to some external substratum (for example the sub-dermal layer in the chick skin) by tethers that may be modelled by a linear spring. Thus the body force in (1.4) is $\underline{F}=-s \underline{u}(\underline{x}, t)$ where $s$ is the positive spring constant. Substituting for $F$ and $\sigma$ into (1.4) gives the force balance equation.

\section{Matrix conservation}

The matrix density satisfies the conservation equation

$$
\partial \rho / \partial t=-\nabla \cdot \underline{J}_{\rho}+\text { matrix secretion by cells }
$$

where $J_{\rho}$ is the matrix flux. The matrix moves only when it is deformed due to cellular traction forces, that is, it moves by convection with velocity $\partial \underline{u} / \partial t$. 
Therefore

$$
\underline{J}_{\rho}=\rho \partial \underline{u} / \partial t
$$

We assume negligible matrix secretion on the time scale we are dealing with. Substituting for $\underline{J}_{\rho}$ into (1.6) gives the matrix conservation equation.

We consider in this paper only the one-dimensional situation and nondimensionalize the model equations (1.1), (1.4) and (1.6) by introducing the quantities

$$
\begin{gathered}
x^{*}=x / L, \quad t^{*}=t / T_{0}, \quad n^{*}=n / N, \quad \rho^{*}=\rho / \rho_{0}, \quad u^{*}=u / L, \quad \lambda^{*}=\lambda N^{2}, \\
\beta^{*}=\beta / L^{2}, \quad s^{*}=s \rho_{0}(1+v) L^{2} / E, \quad D_{1}^{*}=D_{1} T_{0} / L^{2}, \\
\alpha^{*}=\alpha \rho_{0} T_{0} / L^{2}, \quad r^{*}=r N T_{0}, \quad \tau^{*}=\tau \rho_{0} N / E, \quad \mu=\left(\mu_{1}+\mu_{2}\right)(1+\nu) / E T_{0},
\end{gathered}
$$

where $L$ and $T_{0}$ are typical length and time scales respectively, and $\rho_{0}$ is a typical matrix density. The governing equations become, omitting the asterisks for algebraic simplicity,

$$
\begin{gathered}
\partial n / \partial t=D_{1} \partial^{2} n / \partial x^{2}-\partial / \partial x(n \partial u / \partial t)-\alpha \partial / \partial x\{n \partial \rho / \partial x\}+r n(1-n) \\
\partial / \partial x\left[\mu \partial^{2} u / \partial x \partial t+\partial u / \partial x+\left[\tau n /\left(1+\lambda n^{p}\right)\right]\left\{\rho+\beta \partial^{2} \rho / \partial x^{2}\right\}\right]=\operatorname{su} \rho \\
\partial \rho / \partial t+\partial / \partial x\{\rho \partial u / \partial t\}=0
\end{gathered}
$$

These equations were studied in the domain $x \in[0,1]$ with periodic boundary conditions.

In the next section, we discuss the dispersion relation for the linearized system. Setting various parameters equal to zero gives rise to a wide variety of possible dispersion relations suggesting a richness in pattern forming capability of the model. We also describe a method for pattern selection using nonlinear least squares fitting to a desired dispersion relation. In Sect. 3 we briefly describe the numerical technique used to compute solutions to (1.7) and present results of the numerical simulations. We use the results obtained to set up a scenario for sequential formation of dermal aggregates (papillae) in the chick back (dorsal ptylera). In Sect. 4 we consider the model in the light of Davidson's (1983a, b) experiments on the mechanism of feather development in the dorsal pteryla and discuss the possibility of using the nonlinear least squares technique for determining the parameter space wherein a certain mode is selected in general pattern formation problems.

\section{Linear theory of pattern selection}

We consider the stability of the biologically relevant steady state

$$
n=\rho=1, \quad u=0
$$

of (1.7) in the usual way by setting

$$
n=1+n^{\prime}, \quad \rho=1+\rho^{\prime}, \quad u=u^{\prime},
$$


where $\left|n^{\prime}\right|,\left|\rho^{\prime}\right|$ and $\left|u^{\prime}\right|$ are small. Substituting into (1.7) and keeping only first order terms in the primed variables we have a set of linear equations for $n^{\prime}, u^{\prime}$ and $\rho^{\prime}$. We look for solutions of the form

$$
\left(\begin{array}{c}
n^{\prime} \\
u^{\prime} \\
\rho^{\prime}
\end{array}\right) \propto \exp [\sigma(k) t+i k x] .
$$

The solvability condition gives rise to the dispersion relation

$$
\sigma\left[\mu k^{2} \sigma^{2}+b(k) \sigma+c(k)\right]=0
$$

where

$$
\begin{aligned}
b(k)= & {\left[\mu D_{1}+\beta \tau /(1+\lambda)\right] k^{4}+\left[1+\mu r-2 \tau /(1+\lambda)^{2}\right] k^{2}+s, } \\
c(k)= & \left\{\tau \beta D_{1} /(1+\lambda)\right\} k^{6}+\left\{\tau(1+\lambda)^{-1}\left[r \beta-D_{1}-\alpha(1-2 \lambda /(1+\lambda))\right]+D_{1}\right\} k^{4} \\
& +\left[s D_{1}+r-r \tau /(1+\lambda)\right] k^{2}+r s .
\end{aligned}
$$

Here we have taken the inhibition parameter $p=2$ (see $(1.7 \mathrm{~b})$ ). When solved this equation gives the growth rate $\sigma$ as a function of the parameters and the wave number, $k$.

Setting different parameters to zero gives rise to a wide selection of dispersion relations (Murray and Oster (1984a, b); Maini et al. (1984)). The steady state (2.1) will be unstable to spatial perturbations of wave number, $k$, if the growth rate for that $k$ is positive; that is, where $\operatorname{Re} \sigma(k)>0$. On the domain $[0,1]$, the steady state (2.1) will then be unstable if there exists an integer $n$, such that $\operatorname{Re} \sigma(2 n \pi)>0$. To isolate the $n$th mode, we need to choose parameters such that $\operatorname{Re} \sigma(2 n \pi)>0$ and $\operatorname{Re} \sigma(2 m \pi)<0$ for all other integers $m$.

Clearly the complicated form of the dispersion relation (2.2) makes the determination of the parameter space in which a certain mode is isolated very difficult. We approach this by solving the inverse problem, that is, we choose the form of the dispersion relation we would like, then fit the actual dispersion relation to it using a nonlinear least squares package. The routine we used is based on the Levenberg-Marquardt algorithm (More (1977)) and minimizes the sum of squares of a set of nonlinear functions in $n$ variables. Thus, if we choose the functional form, $y$, of the dispersion relation required to isolate a particular mode, the package chooses values of the parameters $\left\{D_{1}, \alpha, r, \mu, \tau, \beta, s, \lambda\right\}$ which minimizes the objective function

$$
J=\sum_{i=1}^{m}|\operatorname{Re} \sigma(i)-y(i)|^{2}
$$

that is, it fits $\operatorname{Re} \sigma$ to the "idealized" dispersion form $y$. It is not necessary to choose a functional form for $y$, we can simply choose $m$ fictitious "data" points that mimic the desired shape of the dispersion relation and minimize (2.3). The best fit may involve negative values of some of the parameters and these values have no meaning in a biological context. To overcome this problem, we replace each parameter, $x_{1}$ by $f\left(p_{1}\right)$, where $f\left(p_{i}\right) \geqslant 0$, and then minimize $J^{\prime}\left(p_{1}, \ldots, p_{n}\right)=$ $J\left(f\left(p_{1}\right), \ldots, f\left(p_{n}\right)\right.$ with respect to $p_{i}$. For our simulations we chose $x_{i}=\exp \left(p_{i}\right)$. Thus even if $p_{i}$ is negative, the biological parameter $x_{i}$ is positive. The exponential 


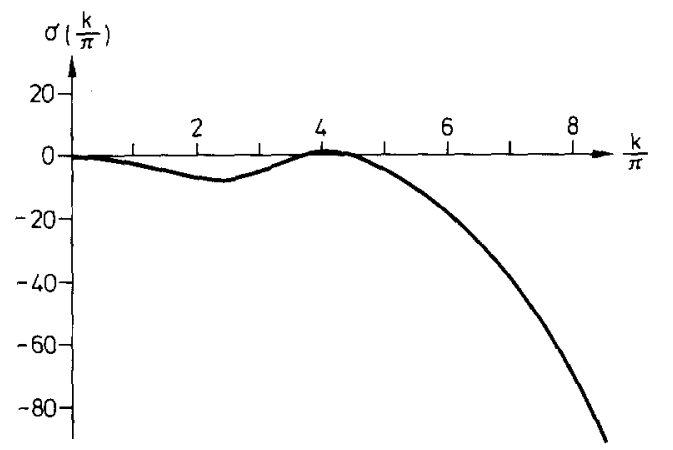

Fig. 2.1. Illustration of the curve fitting technique to isolate the $k / \pi=4$ mode. We choose data points such that this mode is the only unstable permissible mode. The curve fitting routine fits $\operatorname{Re} \sigma(k)$ to these points. The package used minimises the sum of squares of $m$ nonlinear functions in $n$ variables and is based on the LevenbergMarquardt algorithm (Moré (1977)). We took $m=8, n=7$, that is, we set $r=0$ and fitted for the remaining seven parameters. The values of the parameters here are $D_{1}=$ $0.2, s=177.8, \quad \beta=0.0021, \quad \tau=10.2, \mu=$ $0.022, \lambda=1.96, \alpha=0.026$. Computations were performed on the CRAY1 and CDC 7600 at Los Alamos National Laboratory

has the disadvantage that small changes in $p_{i}$ lead to large changes in $x_{i}$. Because only certain ranges of parameter values make biological sense, good initial parameter guesses and/or choices of fictitious data points, were required to keep the final values within the relevant range. Absolute minima of the objective function were not required, only sets of parameters that gave rise to dispersion relations of the required form. Using this criterion multiple solutions could be found by different choices of initial parameters or data values. For this problem, in which a lot of earlier analysis had been done, good initial guesses were easily found which yielded biologically reasonable final parameter values. Figure 2.1 illustrates the dispersion relation thus obtained to isolate the $k / \pi=4$ mode. For other problems, different choices for $f\left(p_{i}\right)$ might be more appropriate. One referee suggested $f\left(p_{i}\right)=p_{i}^{2}$, although this has the disadvantage of generating a singular Jacobian matrix when $p_{i}=0$, that is $\partial J / \partial p_{i}=2 p_{i} \partial J / \partial x_{i}$. Algorithms other than Levenberg-Marquardt in which constraints could be placed on the parameter search domain could also be tried.

In some cases, depending on the values we choose for the "data points" $y$, the best fit obtained was of the form illustrated in Fig. 2.2. However, since traction is a destabilizing factor, increasing $\tau$ moves the dispersion relation upwards. The

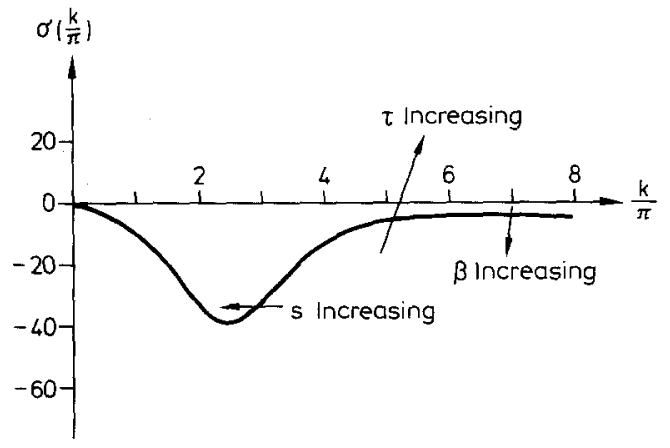

Fig. 2.2. In some cases the curve fitting routine was unable to give the shape of the dispersion relation required to isolate a mode. However, we know from physical considerations that $\tau$ moves the dispersion relation vertically, $\beta$ controls the stability of higher wave number spatial variation and $s$ controls the stability of lower wave number spatial variation. By varying these three parameters we can convert this type of dispersion relation into one that isolates a mode (as illustrated in Fig. 2.1). Thus the curve fitting routine has reduced the parameter search from a seven-dimensional space to a three-dimensional space 
parameter $\beta$ measures long range traction and affects the stability of large wave number spatial variations while varying $s$, a measure of the elastic (short range) tethering force, affects the stability of small wave number spatial variations. Thus, given a dispersion relation of the form illustrated in Fig. 2.2, we can vary these three parameters to isolate a particular mode.

Although this is a rather crude technique, in view of the large number of parameters and the complexity of the dispersion relation, the fitting procedure worked quite well. Occasionally the minimization procedure was trapped in a local minima not of the prescribed form (for example, Fig. 2.2). Nevertheless, the procedure reduced the problem from a seven-dimensional parameter space search to a three-dimensional one (in almost all of these calculations we neglected cell division setting $r=0$ ). By varying only $\tau, \beta$ and $s$ and keeping the other parameters fixed at the values given by the fitting routine, it was possible to isolate modes.

\section{Numerical study of nonlinear pattern selection}

\section{(i) Numerical technique}

We numerically solved (1.7) with $r=0$, periodic boundary conditions, and initial conditions corresponding to $u$ and $\rho$ at their uniform steady state, $u(x, 0)=0$ and $\rho(x, 0)=1$, and $n$ randomly perturbed from its steady state, that is $n(x, 0)=$ $1+\delta(x)$, where $\delta(x)$ was picked from a uniform distribution and took values between -0.05 and 0.05 . To do this we used a general purpose PDE solver written by J. M. Hyman called PDE1D, currently available only at the Los Alamos National Laboratory. The program uses the method of lines to solve systems of initial-boundary-value partial differential equations in one space dimension, and is an updated, expanded and modified version of the publicly available program MOL1D (Hyman (1979)). Because PDE1D and its higher dimensional analogs PDE2D and PDE3D are still under development we do not discuss the panoply of features available in the code.

Equation (1.7b) was not of the general form required by the package, that is

$$
\underline{u}_{t}=g\left(x, t, \underline{u}, \underline{u}_{x}, \underline{u}_{x x}, \ldots\right),
$$

since it contained the derivative $u_{x x t}$. The spatial part of all derivatives were represented using a three point, second order finite difference method. For the $u_{x x t}$ equation this difference matrix was inverted on each time step to explicitly define $u_{t}$. The resulting system of ordinary differential equations in time were solved by an iterated Runge Kutta Method (RKM). The iterated RKM uses a nested sequence of Runge Kutta methods of increasing orders to achieve an accuracy of $10^{-7}$ per time step. If the error in the solution is greater than the imposed tolerance, the method recalculates the solution using a higher order (more accurate) RKM. The procedure is continued until the tolerance criterion is satisfied (see Hyman 1980 for further details).

The reliability of the numerical method was monitored in various ways. The solutions were occasionally recalculated in finer meshes and/or with fourth or sixth order finite differences. The integration in time was also carried out using Adams-Bashforth-Moulton and Backward Difference Linear Multistep 
integration methods. Different, but equivalent, formulations of the equations were also solved to ensure the numerical approximations had converged. There were no constraints in the method to ensure that the total cell number and total matrix density remained constant. Conservation conditions were monitored to again check the accuracy of the method.

\section{(ii) Mode selection}

Figure 3.1 illustrates the results obtained on solving (1.7) using different sets of parameters that isolate modes one, two, three and four respectively.

If we choose parameters such that the dispersion relation is complex, linear analysis predicts a temporally varying solution (travelling waves). Figure 3.2 illustrates the result of simulating the full nonlinear system (1.7) with parameters such that $\operatorname{Re} \sigma(2 \pi)>0, \operatorname{Re} \sigma(2 m \pi)<0$ for all other integers, $m ; \operatorname{Im} \sigma(2 \pi) \neq 0$.

These simulations clearly illustrate the importance of linear analysis in selecting parameter spaces wherein spatial patterns may form. Linear analysis, of course, predicts unbounded temporal growth, and it is the nonlinear terms that limit this unbounded growth and lead to a steady state. Figure 3.3 illustrates two cases in which the nonlinearities cannot bound the growth. In Fig. 3.3a the values of the parameters are identical to those in Fig. 3.1a except that $\lambda$, the contact inhibition parameter, is set to zero. Thus the cell traction increases linearily as cell density increases. Once an aggregate is formed, the traction exerted by the cells in the aggregate will continue to recruit cells from the surrounding neighbourhood leading to the formation of a delta function in cell density. This has important biological implications in that it indicates the necessity of cell-cell contact inhibition for stable spatial structures.

In several of the computations, the values of $D_{1}$ and $\alpha$ are very small, and $r=0$. Thus the cell density equation is virtually identical to the matrix density equation - convection is the major form of motion. Therefore we would expect $n$ and $\rho$ to be similar. This is clearly illustrated by our results. Thus the stress due to cell traction may be approximated by

$$
\boldsymbol{\sigma}_{\text {cell-matrix }}=\left[\beta_{1} \tau n /\left(1+\lambda n^{p}\right)\right]\left[n+\beta \partial^{2} n / \partial x^{2}\right]
$$

where $\beta_{1}$ is some positive constant. If we choose $p=2$, the first term in $\sigma_{\text {cell-matrix }}$ asymptotes to $\beta_{1} \tau / \lambda$ for $n$ large, and $\beta \tau n^{2}$ for $n$ small. Thus, once an aggregate is formed, it will continue to exert a constant traction, while the (small) population of cells nearby exert a traction proportional to the square of the cell density, which is small. This would lead to unbounded growth in the cell aggregate, unless the value of $\partial^{2} n / \partial x^{2}$ is such that the contribution of this term to $\sigma_{\text {cell-matrix }}$ balances the above variation in traction. In Fig. $3.3 \mathrm{~b}$ this clearly does not happen. If we increased $p$, then, for large cell density, the cell traction, which behaves like $n^{2-p}$ for large $n$, would be small and a bounded steady state may exist (Fig. 3.3c).

\section{(iii) Fast mode selection}

In Sect. 2 we mentioned that the dispersion relation gave rise to a wide variety of behaviours depending on the values of the parameters. On setting certain parameters to zero, there is the possibility of an infinite linear growth rate for a particular mode and we would expect fast selection for this mode (Maini et al. 

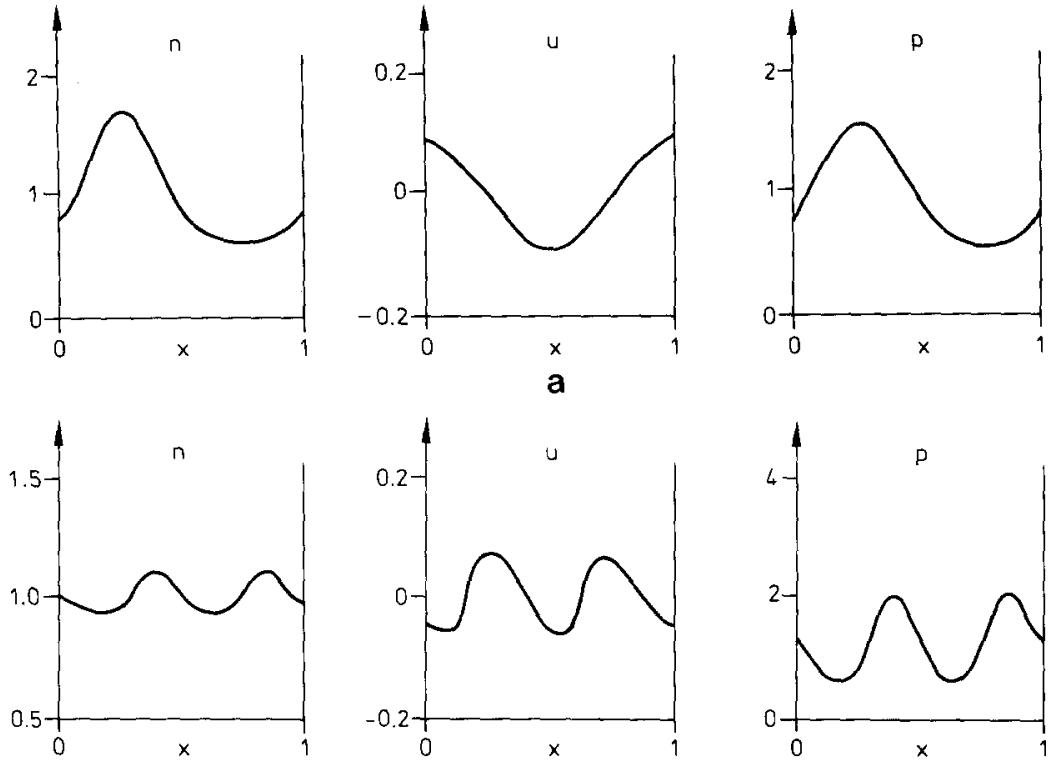

b
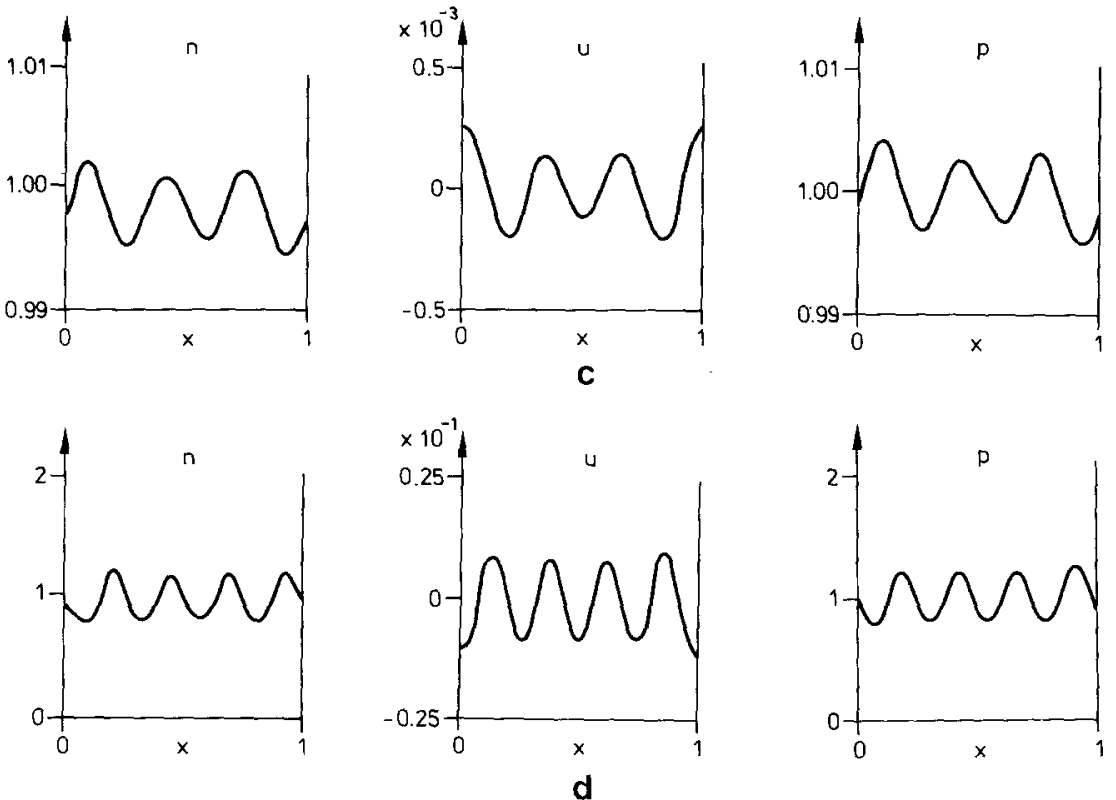

Fig. 3.1a-d. Steady state solutions for (1.7) in one dimension with $r=0$. In this case, the parameters were chosen to isolate a particular mode as described in Sect. 2. In all these simulations the tolerance for the time integration was $10^{-7}$. a Linear analysis predicts one aggregate. Parameters: $D_{1}=\alpha=0.001$, $\lambda=0.12, \beta=0.015, \tau=2.3, s=57.32$ and $\mu=1.0$. Number of grid points $($ ngrd $)=21$, time $(\mathrm{t})=70$. b Linear analysis predicts two aggregates. Parameters: $D_{1}=0.2, \alpha=0.026, \lambda=1.96, \beta=0.0021$, $\tau=10.2, s=177.8, \mu=0.022$, ngrd $=81$ and $t=15$. c Linear analysis predicts three aggregates. Parameters: $D_{1}=\alpha=0.001, \lambda=0.12, \beta=0.001, \tau=1.01, s=100.0, \mu=1.0$, ngrd $=81$ and $t=10$. d Linear analysis predicts four aggregates. Parameters: $D_{1}=\alpha=0.001, \lambda=0.12, \beta=0.001, \tau=1.65$, $s=400.0, \mu=1.0$, ngrd $=81$ and $t=200$ 

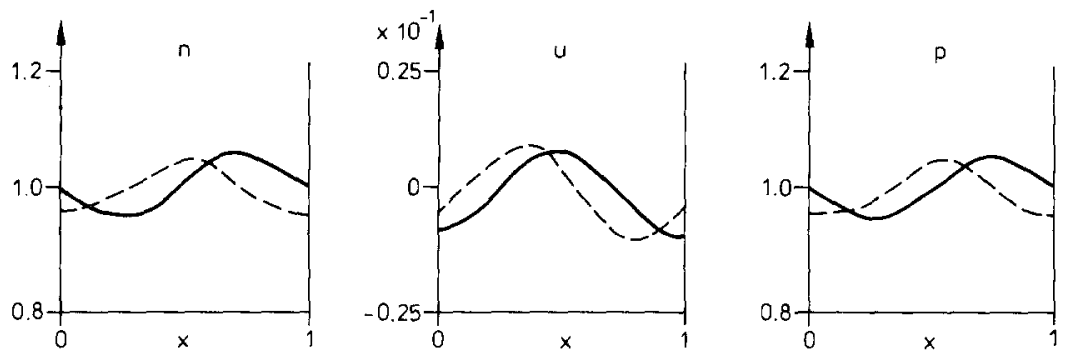

Fig. 3.2. Temporally oscillating solution for (1.7) in one dimension with $r=0, D_{1}=0.015, \alpha=0.002$, $\lambda=0.5, \beta=0.006, \tau=2.4, s=28.69, \mu=0.01$ and $n g r d=41$. In this case, $\operatorname{Re} \sigma(2 \pi)>0, \operatorname{Im} \sigma(2 \pi) \neq 0$. $-t=0.6,--t=0.85$

(1984)). Of course, by making some of the parameters small instead of zero it is possible to bound the growth rate, but, even so, the maximum (linear) growth rate is still large. To numerically simulate the full nonlinear system for such parameter values is difficult because of stiffness problems. Figure 3.4 illustrates such a dispersion relation and the result of simulating (1.7) with these parameter values. Clearly the first mode (one aggregate) is selected almost immediately and grows until the matrix density goes negative, which is, of course, a reflection of accumulating numerical errors.

\section{(iv) Multiple mode selection}

In this paper we have been concentrated on a single mode selection: we chose parameters such that linear analysis predicted only one permissible unstable mode. An interesting problem is to consider the situation where more than one mode is unstable and to investigate what mode, if any, will be selected.

\section{(v) Sequential formation of two-dimensional patterns}

One of the motivations for studying the ability of the model to generate pattern sequentially comes from the results of Davidson's (1983) experiments on dermal cell aggregation. Leaving aside the controversial problem of which aggregate forms first - the epidermal placode or the dermal papilla - it appears that the first dermal aggregate forms approximately in the centre of the dorsal midline on the chick back. Two further aggregates then form, one on either side of this initial aggregation, also along the dorsal midline. More aggregates then form on either side of the dorsal midline but displaced longitudinally from those on the dorsal midline to give an hexagonal pattern of papillae. The "wave" of aggregations then spreads out forming a regular hexagonal pattern. Although the initial row is not necessary for the formation of subsequent neighbouring rows it does determine the position of the subsequent papillae.

As cells mature, their mechanical properties change. For example, the traction exerted by mesenchymal cells in vitro can increase dramatically over a period of a few days (Harris, personal communication). Suppose that in a given domain the cell tractions are as shown in Fig. 3.5a; that is, the central cells have aged to the point where they have commenced to contract with a force $\tau$, while the peripheral cells have not yet matured to the contraction stage. Then, as shown 

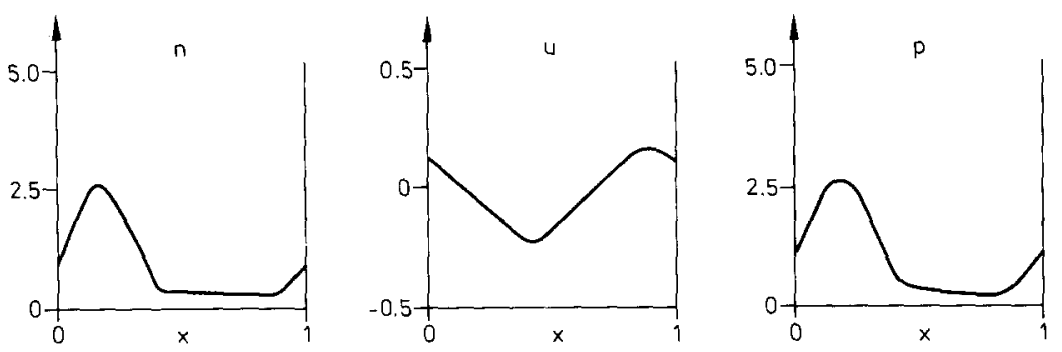

a
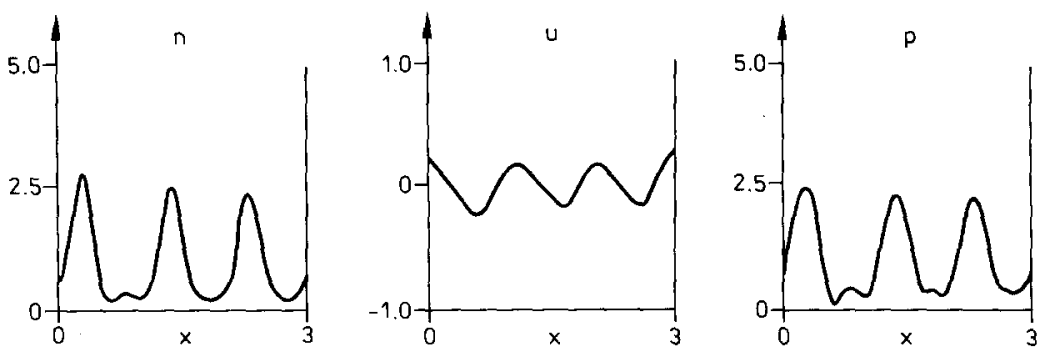

b
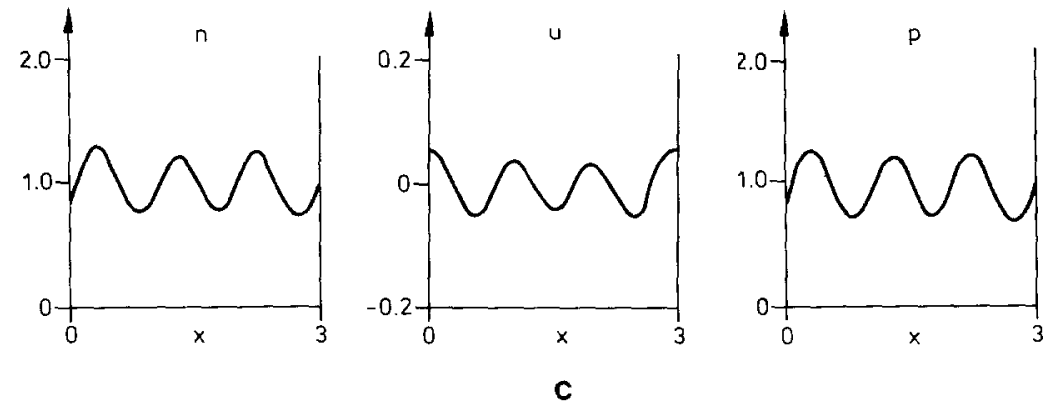

Fig. 3.3. a Linear analysis predicts one aggregate. The parameters are identical to those in Fig. 3.1a except that $\lambda=0$. Thus there is no cell-cell contact inhibition and the traction exerted by the growing aggregation pulls surrounding cells into the aggregate leading to a delta function in cell density, $n$. b Linear analysis predicts the formation of one aggregate on the unit interval. In this case, there is effectively no contact inhibition (see text for detail). Parameters are $D_{1}=\alpha=0.001, \lambda=0.12, \beta=0.015$, $\tau=3.0, s=57.32, \mu=1.0, n g r d=101$ and $t=10$. In this case, traction/cell $(\tau(n))=\tau n /\left(1+\lambda n^{4}\right)$ and contact inhibition is strong enough to bound the cell aggregates (see text for detail). Parameters are identical to (a) except $\lambda=0.1, n g r d=31$. The result is shown for time $t=1000$

in Fig. 3.5c, a single, centrally located aggregation of cells will accumulate. If this aggregation is stabilized by subsequent developmental processes, then as the peripheral cells mature and commence contracting an additional two peaks will appear as shown in Fig. 3.5c. In this way, a single peak will lead to three equally spaced aggregates. Thus the model can explain the formation of the initial row of feather germs. 
536

A. S. Perelson et al

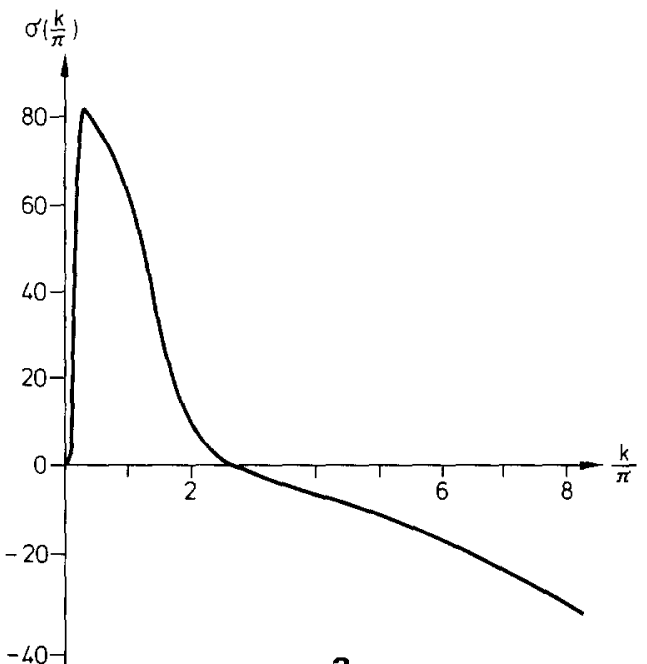

a
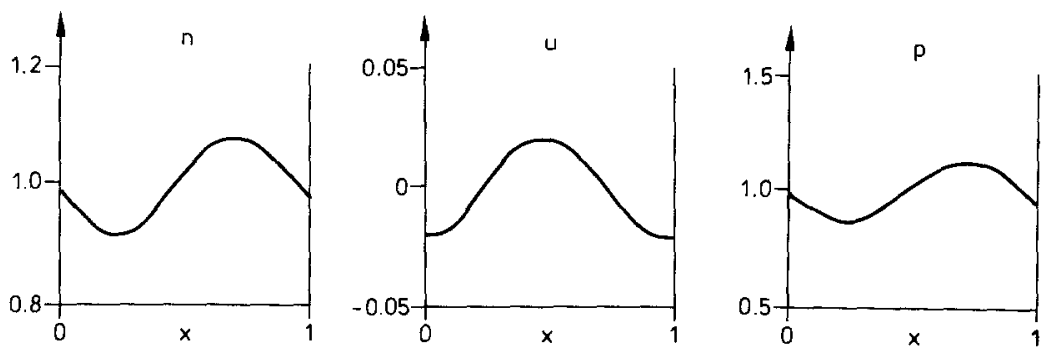

b
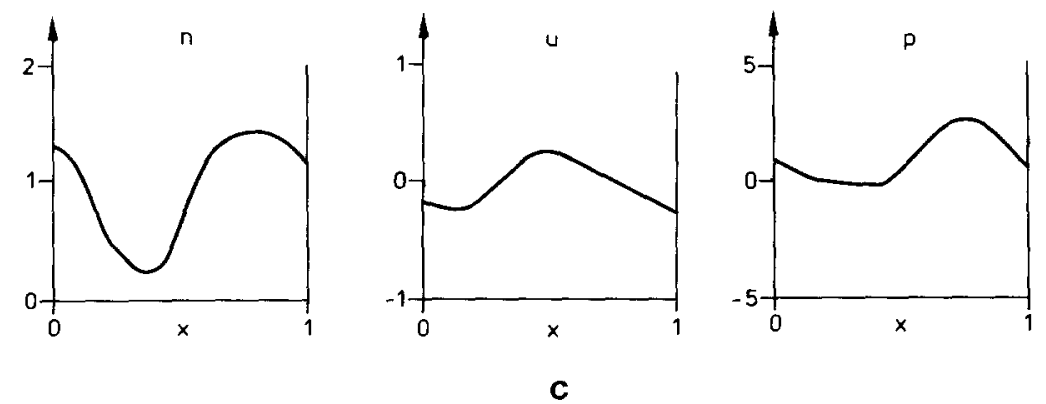


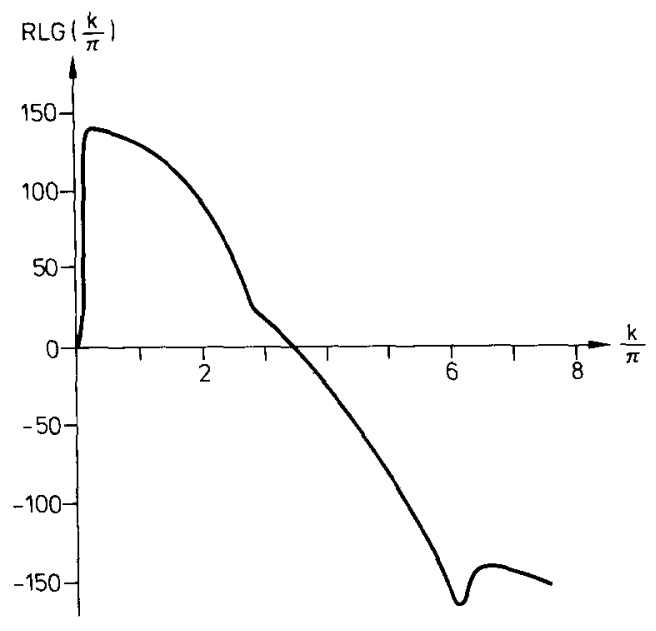

d
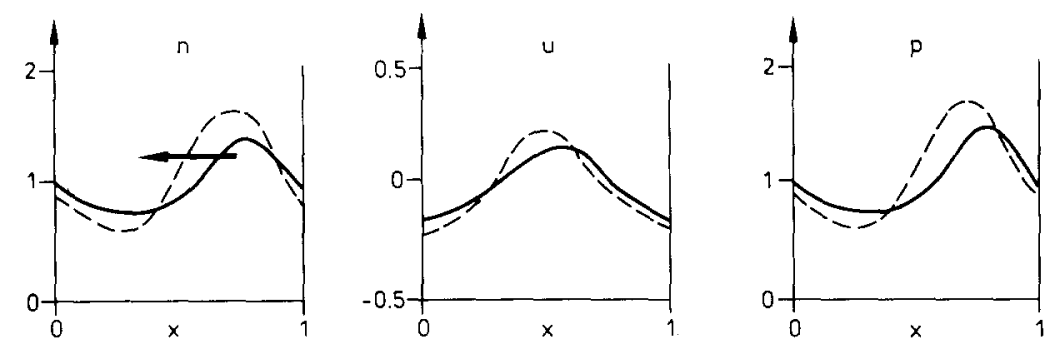

e

Fig. 3.4a-e. Fast mode selection. a Dispersion relation for parameters $p=4, D_{1}=0.05, \alpha=0.0$, $\lambda=0.5, \beta=0.01, \tau=4.0, s=0.0, \mu=0.01, r=1.0$. b Result of simulation at $t=0.5 \quad(n g r d=21$ ). Clearly the first mode has been selected very quickly. c At $t=1.2$, the matrix density, $\rho$, goes negative. d With parameters $D_{1}=0.2, \quad r=1.0, \quad \beta=0.01, \quad \tau=1.2, \quad \mu=0.01, \quad p=2, \quad \lambda=0.0=s=\alpha$, the real part of the dispersion relation is qualitatively similar to a, but now there is an imaginary part such that $\operatorname{Im} \sigma(2 / \pi) \neq 0$. In this case we have a growing, travelling aggregate e (number of grid points $=101$ ). These simulations encountered stiffness difficulties and took roughly $1 / 2$ million time steps and over 30 minutes CRAY time

The simulations in Fig. 3.6a, b provide strong evidence for the idea that this initial row sets up a strain field which guides cell aggregations in the secondary rows to occur at locations between the original aggregations. Figure 3.6c illustrates how such a scenario could lead to two-dimensional hexagonal patterns. Simulations of the model in two spatial dimensions could be used to establish this more rigorously.

\section{Discussion}

In previous publications (Oster et al. (1983), Murray and Oster (1984a, b), Maini et al. (1984)) we performed a linear study of the mechanical model described in 


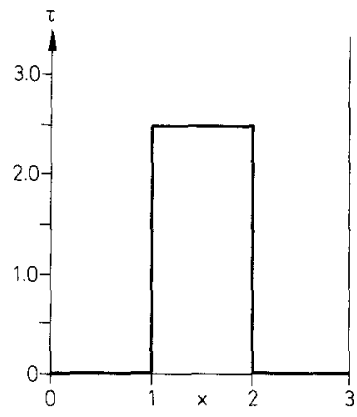

a

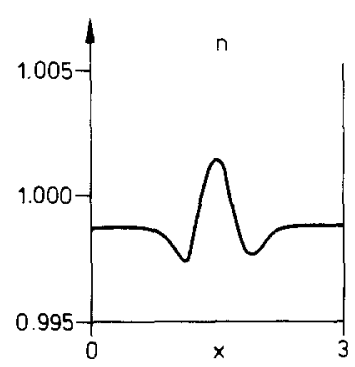

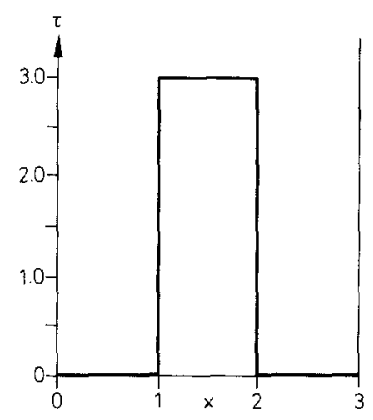

b

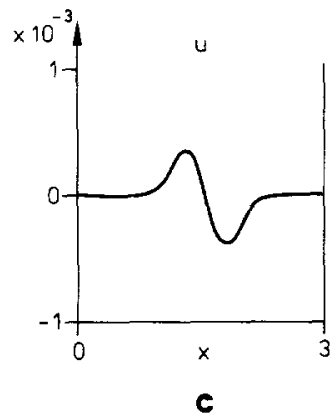

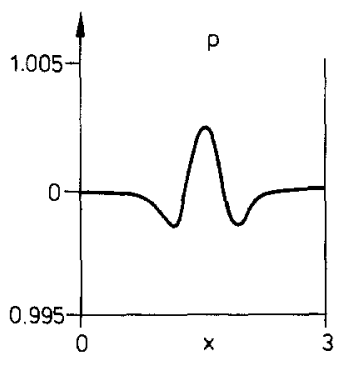

Fig. 3.5a-c. Sequential pattern formation. With parameters $D_{1}=\alpha=0.001, \lambda=0.12, \beta=0.015, \mu=1.0$, $s=57.32, r=0, p=2$, linear analysis predicts one aggregate on the unit interval. We considered the interval $[0,3]$ with a spatially varying $\tau$ a. At $t=300$, the uniform steady state is stable. If we increase $\tau$ on $[1,2], \mathbf{b}$ the homogeneous steady state in $n$ with initial random perturbations bifurcates to one aggregate at $t=1000 \mathrm{c}$, (number of grid points $=101$ ). If we set $\tau$ to 3.0 on $[0,3]$ and $p=4$, we get three aggregates (see Fig. 3.3c)

Sect. 1 which led us to predictions on the pattern forming capabilities of the mechanism. In this paper we have established the nonlinear pattern forming potentialities of the model. Although our calculations are one-dimensional, they provide considerable confidence that the model can generate regular stable two-dimensional patterns. We have presented a scenario for the formation of dermal papillae which is consistent with the results of Davidson's (1983) experiments on feather germ pattern formation. The formation of the initial row of papillae is based on the cell traction being spatially inhomogeneous, a fact amenable to experimental verification.

In Sect. 3 we presented only a partial investigation into the pattern forming capabilities of the model. We neglected cell division in most cases and ignored the possibilities of long range diffusion. The wide variety of dispersion relations, together with the nine-dimensional parameter space of the full model, suggest a richness of pattern forming capability of such a mechanism.

Using nonlinear least squares fitting to the desired dispersion relation greatly simplified the search of our restricted seven-dimensional parameter space. Our experience with this technique leads us to believe that nonlinear least squares or maximum likelihood fitting to a desired dispersion relation should prove useful 

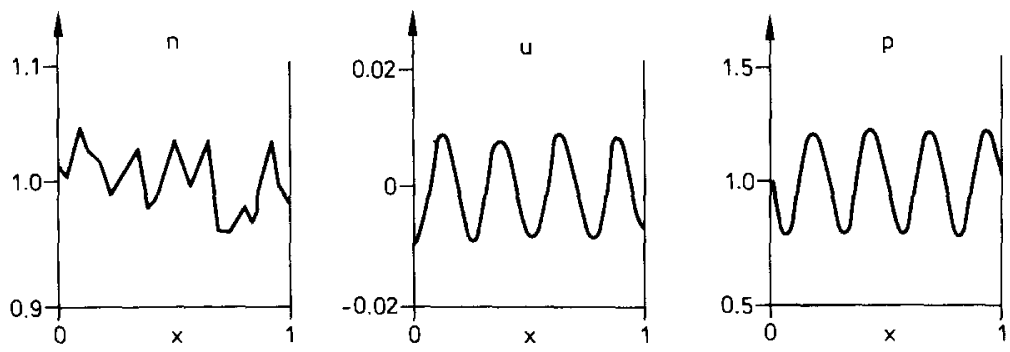

a
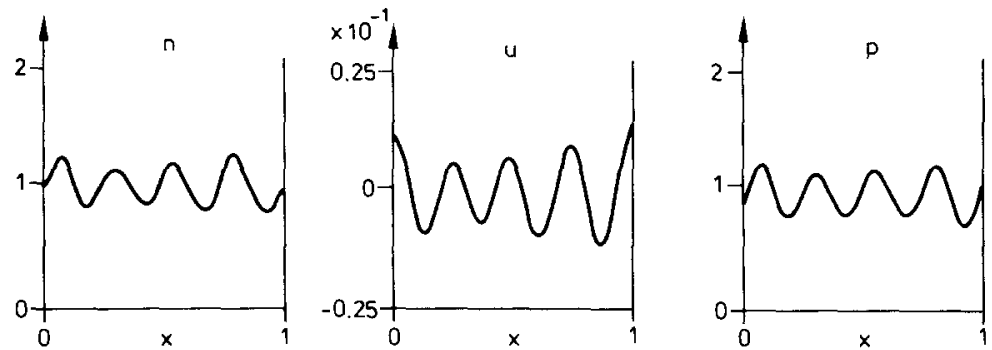

b

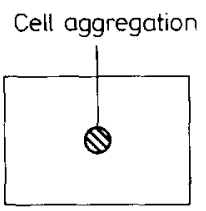

(ii)

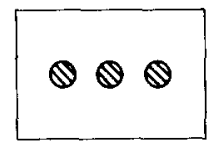

(iii)

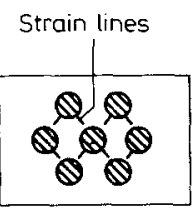

(iv)

$\mathbf{C}$

Fig. 3.6. a Initial conditions: random perturbations in cell density, $n$; matrix displacement, $u$, and matrix density, $\rho$, correspond to Fig. $3.1 \mathrm{~d}$, that is, we have a pre-strained matrix. b The steady state solution at $t=10$ with $n g r d=101$ and model parameters identical to Fig. $3.1 \mathrm{~d}$. Note that the aggregates are $180^{\circ}$ out of phase with those in Fig. 3.1d. c Conjectured scenario for hexagonal pattern formation in an idealised section of chick back using the above and Fig. 3.5: as a spatially varying cell traction increases, the uniform cell density, (i), becomes unstable and evolves into an isolated aggregation (ii); this evolves in a single row of aggregates as traction increases either side of the initial aggregate (iii) and sets up a strain field causing condensations along a neighbouring row at intermediate points; (iv) illustrates how this leads to a hexagonal array of dermal cell aggregations

in other pattern selection problems, especially those involving high dimension parameter spaces. Picking fictitious data points to indicate the shape of the desired dispersion relation was clearly imprecise and not always successful. Many choices could be made which gave rise to the desired pattern, although not all were handled equally well by the Levenberg-Marquardt algorithm we employed. Mapping out the complete regions of parameter space which correspond to a 
particular pattern remains a challenging problem. Murray (1982) investigated this problem with respect to reaction diffusion mechanisms which generate spatial patterns. Perhaps parameter estimation techniques will provide a window through which one can gain insights into this problem in general.

Acknowledgments. Portions of this work were performed under the auspices of the U.S. Department of Energy at the Los Alamos National Laboratory and other portions at the Centre for Mathematical Biology, University of Oxford.

A.S.P. and G.F.O. would like to acknowledge the support of the Centre for Mathematical Biology, University of Oxford and the Science and Engineering Research Council of Great Britain (Grant GR/D/13573).

J.D.M. would like to acknowledge the support of the Center for Nonlinear Studies, Los Alamos National Laboratory, where much of this work was carried out as the Stan Ulam Visiting Scholar.

P.K.M. would like to thank the Center for Nonlinear Studies, Los Alamos National Laboratory for support during a visit and the Science and Engineering Research Council of Great Britain for a postdoctoral assistantship (Grant GR/D/13573).

G.F.O. would also like to acknowledge the support of the U.S. National Science Foundation, Grant MCS-8110557.

\section{References}

Davidson, D.: The mechanism of feather pattern development in the chick I. The time of determination of feather position. J. Embryol. Exp. Morph. 74, 245-259 (1983a)

Davidson, D.: The mechanism of feather pattern development in the chick II. Control of the sequence of pattern formation. J. Embryol. Exp. Morph. 74, 261-273 (1983b)

Dhouailly, D.: Early events in retinoic acid-induced ptilopody in the chick embryo. Roux's Arch. Dev. Biol. 192, 21-73 (1983)

Gierer, A., Meinhardt, H.: A theory of biological pattern formation. Kybernetik 12, 30-39 (1972)

Harris, A. K., Ward, P., Stopak, D.: Silicone rubber substrata: a new wrinkle in the study of cell locomotion. Science 208, 177-179 (1980)

Hyman, J. M.: MOL1D: A general purpose subroutine package for the numerical solution of partial differential equations. Manual, Los Alamos Nat. Lab., LA-7595-M (1979)

Hyman, J. M.: Numerical methods for nonlinear differential equations. In: Bishop, A. R., Campbell, D. K., Nicolaenko, B. (eds.). Nonlinear problems: present and future, pp. 91-107. Amsterdam: North Holland 1980

Maden, M.: Vitamin A and pattern formation in the regenerating limb. Nature 295, 672-675 (1982)

Maini, P. K., Murray, J. D., Oster, G. F.: A mechanical model for biological pattern formation: a nonlinear bifurcation analysis. In: Sleeman, B. D., Jarvis, R. J. (eds.). Ordinary and partial differential equations. Proceedings, Dundee 1984 (Lect. Notes Math., vol. 1151, pp. 252-269), Berlin Heidelberg New York: Springer (1984)

Meinhardt, H.: Models of biological pattern formation. London: Academic Press 1983

Moré, J. J.: The Levenberg-Marquardt algorithm: implementation and theory. In: Watson, G. A. (ed.). Numerical Analysis. Proceedings, Dundee 1977 (Lect. Notes Math., vol. 630, pp. 105-116), Berlin Heidelberg New York: Springer (1978)

Murray, J. D.: Non-linear-differential-equation models in biology. Oxford: Clarendon Press 1977

Murray, J. D.: A pre-pattern formation mechanism for animal coat markings. J. Theor. Biol. 88, $161-199(1981)$

Murray, J. D.: Parameter space for Turing instability in reaction-diffusion mechanisms: a comparison of models. J. Theor. Biol. 98, 143-163 (1982)

Murray, J. D., Oster, G. F.: Generation of biological pattern and form. IMA J. Math. Appl. Med. Biol. 1, 51-75 (1984a)

Murray, J. D., Oster, G. F.: Cell traction models for generating pattern and form in morphogenesis. J. Math. Biol. 19, 265-279 (1984b)

Oster, G. F., Murray, J. D., Harris, A. K.: Mechanical aspects of mesenchymal morphogenesis. J. Embryol. Exp. Morph. 78, 83-125 (1983) 
Oster, G. F., Murray, J. D., Maini, P. K.: A model for chondrogenic condensations in the developing limb: the role of the extracellular matrix and cell tractions. J. Embryol. Exp. Morph. 89, 93-112 (1985)

Purcell, E.: Life at low Reynolds number. Am. J. Phys. 45, 1-11 (1977)

Sengel, P.: Morphogenesis of skin. Develop. Cell Biol. Ser. 3. Cambridge: Cambridge Univ. Press 1976

Shubin, N., Alberch, P.: A morphogenetic approach to the origin and basic organisation of the tetrapod limb. In: Hecht, M., Wallace, B., Steere, W. (eds.). 20. New York: Plenum Press 1986

Solursh, M.: Cell-cell interactions and chondrogenesis. In: Hall, B. K. (ed.). Cartilage 2: development, differentiation and growth. New York: Academic Press 1983

Turing, M. A.: The chemical basis of morphogenesis. Philos. Trans. Roy. Soc. (Lond.) B237, 37-73 (1952)

Wolpert, L.: Positional information and pattern formation. Philos. Trans. Roy. Soc. (Lond.) B295, 441-450 (1981)

Received April 17/Revised July 7, 1986 